\title{
Algoritmo para paramentação, esparamentação e prevenção de lesões faciais: covid-19
}

\section{Algorithm for paramentation, deparamentation and prevention of facial injuries: covid-19}

\section{Geraldo Magela Salomé (1)}

Universidade do Vale do Sapucaí (Pouso Alegre). Minas Gerais, Brasil. salomereiki@univas.edu.br

\begin{abstract}
RESUMO | OBJETIVO: Elaborar algoritmos para orientar os profissionais da saúde na correta utilização dos Equipamentos de Proteção Individual e oferecer medidas preventivas relacionadas às lesões de pele facial causadas pelo uso inadequado dos equipamentos de proteção individual durante a pandemia da SARS-CoV-2. MÉTODO: Revisão integrativa da literatura junto às bases de dados das Ciências da Saúde, MEDLINE, SciELO e LILACS, no período de 2015 a 2020, sendo utilizados para a busca os descritores: COVID-19, Lesão por pressão relacionada a dispositivo médico e Equipamentos e provisões. RESULTADOS: Foram desenvolvidos três algoritmos que descrevem as técnicas de paramentação, de desparamentação dos equipamentos de proteção individual e as medidas preventivas para evitar as lesões faciais causadas pelo uso inadequado dos equipamentos de proteção individual. CONCLUSÃO: Os algoritmos construídos oferecem ao profissional de saúde a descrição de técnicas do uso dos equipamentos de proteção individual e medidas preventivas para lesão facial causada pelo uso dos equipamentos de proteção individual, assim como possibilitam ao profissional uma melhor visualização, praticidade e entendimento do procedimento a ser realizado.
\end{abstract}

DESCRITORES: COVID-19. Equipamento de proteção individual. Equipamentos e provisões.

\begin{abstract}
OBJECTIVE: To develop algorithms to guide health professionals in the correct use of Personal Protective Equipment and to offer preventive measures related to facial skin injuries caused by the inappropriate use of personal protective equipment during the SARS-CoV-2 pandemic. METHOD: Integrative literature review with the databases of Health Sciences, MEDLINE, SCiELO and LILACS, in the period from 2015 to 2020, using the descriptors: COVID-19, Pressure injury related to medical device and Equipment and provisions. RESULTS: Three algorithms have been developed that describe the techniques of dressing, de-dressing of personal protective equipment and preventive measures to avoid facial injuries caused by the inappropriate use of personal protective equipment. CONCLUSION: The algorithms built offer the health professional the description of techniques for the use of personal protective equipment and preventive measures for facial injury caused by the use of personal protective equipment, as well as allowing the professional a better visualization, practicality and understanding of the procedure. to be accomplished.
\end{abstract}

DESCRIPTORS: COVID-19. Personal protective equipment. Equipment and supplies. 


\section{Introdução}

No final do ano de 2019, foi anunciado para o mundo uma nova doença causada pela cepa do coronavírus (SARS-CoV-2), denominada pela Organização Mundial da Saúde (OMS) como COVID-191. Em março de 2020, a Organização Mundial da Saúde classificou a disseminação do vírus como pandemia, com potencial de infectar os seres humanos, tornando-se uma emergência de saúde pública de interesse internacional. ${ }^{1-3}$

O SARS-CoV-2 é altamente transmissível por gotículas e contato, principalmente em locais fechados e ambientes hospitalares. Uma pessoa com infecção pelo novo coronavírus transmite para outras duas ou três pessoas, dependendo das condições ambientais. Locais fechados com pouca ventilação e baixa luminosidade facilitam a transmissão do vírus. Esta taxa de transmissão é chamada número reprodutivo que, na COVID-19, varia entre 2,0 e 3,5.,4,5

Vários profissionais de saúde que estão na linha de frente do COVID-19 estão sofrendo lesões faciais causadas pelo uso do Equipamento de proteção individual (EPIs) e estão sendo infectados pelo COVID-19, sendo importante que as instituições de saúde forneçam a esses profissionais os EPIs, e que eles recebam treinamentos para a técnica da utilização. Tais dispositivos são indispensáveis para contemplar as medidas de precauções padrão, de contato e por gotículas recomendadas no enfrentamento desta pandemia.., 7

Os EPIs são todos os dispositivos de uso individual, destinados a proteger a integridade física do trabaIhador e incluem luvas, protetores oculares ou faciais, protetores respiratórios, aventais e proteção para os membros inferiores, não podendo ser ignorada a higienização das mãos como umas das mais importantes precauções padrão para evitar a contaminação e a disseminação do vírus.

O uso prolongado ou incorreto das máscaras faciais, dos respiradores e dos óculos/viseiras é o responsável pelas forças de fricção e pressão constantes nos tecidos na região da pele facial, levando os profissionais a sofrerem as lesões de pele facial (LPF) e dermatites.., 9 Nesse sentido, é importante a construção de tecnologia educativa, incluindo algoritmo, que ofereça aos profissionais da saúde informações apropriadas sobre as técnicas do uso da EPIs e das ações preventivas e condutas terapêuticas da LPF causada pelos dispositivos. Assim, ao utilizar essa tecnologia corretamente, o profissional previne as lesões e há menos exposição aos agentes infeciosos.

Optou-se por elaborar os algoritmos, pois são mapas gráficos que contribuem para que o profissional possa visualizar e desenvolver passo a passo a técnica do uso dos EPIs e das medidas preventivas da LPF. Algoritmos são constituídos por uma sequência finita de instruções bem definidas realizadas sistematicamente. Eles são empregados comumente no âmbito da saúde; são instrumentos simples, diretos e de fácil acesso, que conferem uma visão completa do processo clínico. São também uma ferramenta indispensável na padronização de técnicas e no gerenciamento da qualidade, constituindo um importante meio de organização de processos que serve de guia para a tomada de decisões. . $^{10,11}$

Este estudo teve como objetivo desenvolver algoritmos para orientar os profissionais da saúde na utilização correta dos EPIs e oferecer medidas preventivas relacionadas às LPF causadas pelo uso inadequado dos EPIs durante a pandemia da SARS-CoV-2.

\section{Método}

Revisão integrativa da literatura aplicada na modalidade de produção de tecnologia, do tipo pesquisa de desenvolvimento metodológico. Delimitaram-se as seguintes etapas para o desenvolvimento da pesquisa: a identificação do tema e seleção da questão de pesquisa; o estabelecimento de critérios para a inclusão e exclusão de estudos; a definição das informações a serem extraídas dos estudos selecionados e categorização dos estudos; a avaliação dos estudos incluídos na revisão integrativa; a interpretação dos resultados, apresentação da revisão; e a síntese do conhecimento. 12

Definiu-se como tema a lesão por pressão facial causada pelo uso inadequado dos EPIs, durante a pandemia da COVID-19, com as seguintes questões norteadoras: Quais são os EPIs e as técnicas corretas de utilização? Quais são as medidas preventivas disponíveis na literatura para evitar a LPF causada pelo uso inadequado dos EPIS durante a pandemia da COVID-19?". 
Para a construção da pergunta adequada para a resolução da questão clínica pesquisada, utilizou-se a estratégia $\mathrm{PICO}^{13}$ - com " $\mathrm{P}$ " correspondendo à população (profissional da saúde); "I" à intervenção (técnica da paramentação e desparamentação dos EPIs, medidas preventivas para LPF); " $\mathrm{C}$ " à comparação (não se aplica, pois esse não é um estudo comparativo) e "O" correspondendo ao desfecho (protocolo em forma de algoritmo).

Foi efetuada uma revisão integrativa da literatura junto às bases de dados das Ciências da Saúde: (MEDLINE), Scientific Eletronic Library Online (SciELO) e Literatura Latino-Americana e do Caribe em Ciências da Saúde (LILACS), com os descritores controlados em Ciências da Saúde: COVID-19, Lesão por pressão relacionada a dispositivo médico e Equipamentos e provisões. A estratégia de busca ocorreu a partir de suas diferentes combinações, utilizando-se o operador booleano AND nos idiomas português, espanhol e inglês, dependendo da base pesquisada.

Para a seleção das publicações, foram adotados como critérios de inclusão: estudos primários com ligação direta com a temática; estar disponível na íntegra, artigos originais e publicados entre 2015 e 2020. Como critérios de exclusão: teses, dissertações, monografias, relatórios técnicos.

A leitura dos títulos e dos resumos foi feita de forma independente pelo autor e por outro pesquisador na área, para assegurar que os textos contemplavam a pergunta norteadora da revisão e atendiam aos critérios de inclusão estabelecidos. Em caso de dúvida a respeito da seleção, optou-se por incluir, inicialmente, a publicação e decidir sobre sua seleção somente após a leitura na íntegra de seu conteúdo.

Para classificar o nível de evidência dos estudos selecionados, foram utilizadas as categorias da Agency for Healthcare Research and Quality ${ }^{14}$, que abrangem seis níveis: Nível 1: evidências resultantes da meta-análise de múltiplos ensaios clínicos controlados e randomizados; Nível 2: evidências obtidas em estudos individuais com delineamento experimental; Nível 3: evidências de estudos quase experimentais; Nível 4: evidências de estudos descritivos (não experimentais) ou abordagem qualitativa; Nível 5: evidências de relatos de caso ou experiência; Nível 6: evidências baseadas em opiniões de especialistas.

A partir desse levantamento, foram construídos os algoritmos que compreendem uma sequência de procedimentos descrita em duas etapas:

Primeira etapa: Descrição da técnica da paramentação e desparamentação dos EPIs utilizados pelos profissionais de saúde durante a pandemia da SARS-CV-2 - Foram identificadas as instruções bem definidas sobre as técnicas da paramentação e desparamentação dos EPIs durante a prática clínica dos profissionais de saúde, que devem ser executadas de forma sistemática com o objetivo de prevenir que os profissionais contraiam a infecção causada pela SARS-Cov-2 e não sejam acometidos por LPF decorrentes do uso inadequado dos EPIs.

Segunda etapa: Lesões causadas por dispositivo provocadas pelo uso inadequado dos EPIs - Foram descritas as medidas preventivas (tipos e técnica da utilização dos dispositivos faciais, técnica da utilização dos cremes, protetores-barreira) que devem ser utilizados para prevenir e tratar as LPF antes durante e depois da utilização dos dispositivos facial.

Por se tratar de uma revisão integrativa da literatura e construção de algoritmo, esse estudo não necessitou da aprovação de Comitê de Ética em Pesquisa, contudo foram considerados aspectos éticos como a citação dos autores dos artigos selecionados.

\section{Resultados}

Identificaram-se, inicialmente, 12.535 artigos; destes, 4.523 foram excluídos por estarem duplicados nas bases de dados. Assim, foram selecionados 8.012 artigos para a leitura do título e 163 para leitura do resumo, que resultou numa amostra de 112 artigos para leitura do texto completo. Deste total, 51 foram excluídos por não responderem à questão norteadora, o que levou ao total de 26 artigos selecionados para construir os algoritmos, conforme exposto na Figura 1. 
Figura 1. Fluxograma do processo de identificação, seleção e inclusão dos estudos, elaborado a partir da recomendação do PRISMA. São Paulo, SP. Brasil, 2020

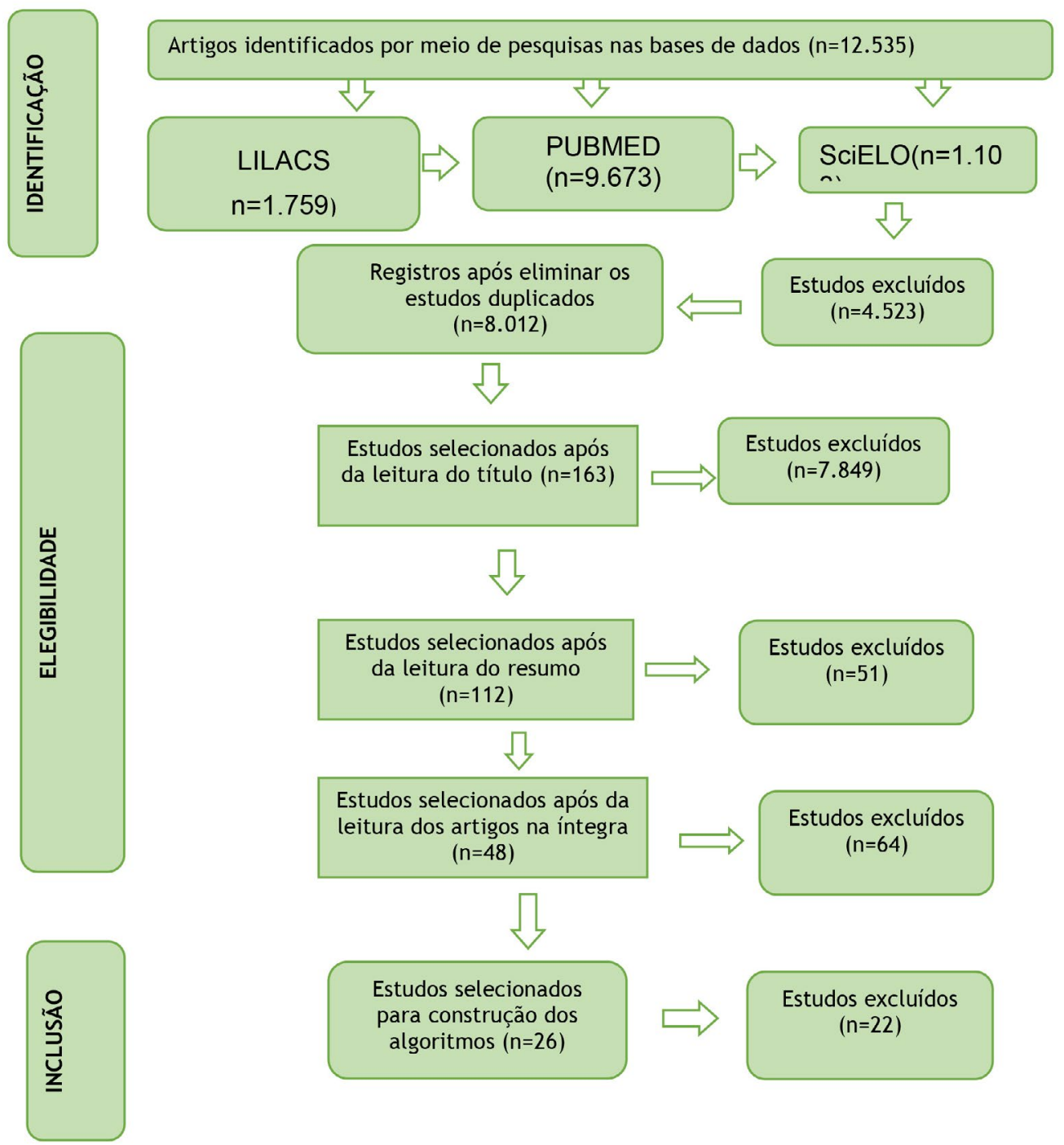

Fonte: Autoria própria. 
No quadro 1, apresentam-se os artigos selecionados durante a revisão integrativa da literatura para desenvolver os algoritmos, os quais foram classificados segundo o nível em evidência.

Quadro 1. Características dos artigos selecionados por meio da revisão integrativa da literatura, para construção dos algoritmos. São Paulo, SP. Brasil, 2020 (continua)

\begin{tabular}{|c|c|c|c|c|c|}
\hline \multicolumn{2}{|r|}{ Autor } & Título & $\begin{array}{l}\text { Periódico/ano/volume } \\
\text { /número }\end{array}$ & Resultados & $\begin{array}{c}\text { Nível de } \\
\text { evidência }\end{array}$ \\
\hline 1 & Black J ${ }^{[15]}$ & $\begin{array}{l}\text { COVID-19 and wound care in the } \\
\text { US }\end{array}$ & $\begin{array}{l}\text { Wounds } \\
\text { International.2020; } \\
11(2): 6-7 . \\
\text { www.woundsinternatio } \\
\text { nal.com }\end{array}$ & $\begin{array}{l}\text { Importância do uso da máscara na } \\
\text { prevenção das lesões de pele facial. }\end{array}$ & 6 \\
\hline 2 & $\begin{array}{l}\text { Taminato } \mathrm{M} \text {, } \\
\text { Mizusaki-Imoto } \\
\text { A, Saconato } \mathrm{H}, \\
\text { Franco ES }{ }^{[16]}\end{array}$ & $\begin{array}{l}\text { N95 respirator associated } \\
\text { pressure ulcer amongst COVID-19 } \\
\text { health care workers }\end{array}$ & $\begin{array}{l}\text { Int Wound J. } \\
\text { 2020;17(5):1525-27. doi: } \\
\text { 10.1111/iwj.13398 }\end{array}$ & $\begin{array}{l}\text { A eficácia do uso da máscara para } \\
\text { prevenir as lesões da pele e de } \\
\text { contrair a infecção durante a } \\
\text { pandemia do COVID-19. }\end{array}$ & 4 \\
\hline 3 & $\begin{array}{l}\text { Taminato M, } \\
\text { Mizusaki-Imoto } \\
\text { A, Saconato H, } \\
\text { Franco EB, Puga } \\
\text { ME, Duarte ML, } \\
\text { et al. }{ }^{[17]}\end{array}$ & $\begin{array}{l}\text { Máscaras de tecido na contenção } \\
\text { de gotículas respiratórias - revisão } \\
\text { sistemática }\end{array}$ & $\begin{array}{l}\text { Acta Paul Enferm. } \\
\text { 2020;33:eAPE20200103. } \\
\text { doi: 10.37689/acta- } \\
\text { ape/2020ar0103 }\end{array}$ & $\begin{array}{l}\text { A máscara é um recurso adicional na } \\
\text { prevenção e deve sempre ser } \\
\text { associada à etiqueta respiratória, } \\
\text { higienização das mãos, } \\
\text { distanciamento social e isolamento } \\
\text { dos casos. }\end{array}$ & 1 \\
\hline 4 & $\begin{array}{l}\text { Gefen A, Ousey } \\
\text { K. }{ }^{[18]}\end{array}$ & $\begin{array}{l}\text { Prevention of skin damage caused } \\
\text { by the protective equipment used } \\
\text { to mitigate COVID-19 }\end{array}$ & $\begin{array}{l}\text { J Wound Care.2020; } \\
\text { 29(6):311. doi: } \\
\text { 10.12968/jowc.2020.29. } \\
6.311\end{array}$ & $\begin{array}{l}\text { Fornece medidas preventivas para } \\
\text { lesão de pele facial causada pelo uso } \\
\text { do EPIs. }\end{array}$ & 6 \\
\hline 5 & $\begin{array}{l}\text { Smart H, } \\
\text { Opinion FB, } \\
\text { Darwich I, } \\
\text { Elnawsany MA, } \\
\text { Kodange C. }{ }^{[19]}\end{array}$ & $\begin{array}{l}\text { Preventing facial pressure injury } \\
\text { for health care providers adhring } \\
\text { to COVID-19 personal protective } \\
\text { equipment requirements }\end{array}$ & $\begin{array}{l}\text { Adv Skin Wound Care } \\
\text { 2020;33(8):418-427. doi: } \\
\text { 10.1097/01.ASW.00006 } \\
69920.94084 . c 1\end{array}$ & $\begin{array}{l}\text { Uso do curativo de silicone para } \\
\text { prevenir lesões na pele facial } \\
\text { causadas por uso do EPIs. }\end{array}$ & 4 \\
\hline 6 & $\begin{array}{l}\text { Alves P, Moura } \\
\text { A, Vaz A, } \\
\text { Ferreira A, } \\
\text { Malcato E, Mota } \\
\text { S, et al. }{ }^{[20]}\end{array}$ & $\begin{array}{l}\text { Recomendação PREPI/COVID19: } \\
\text { Prevenção de lesões cutâneas } \\
\text { causadas pelos Equipamentos de } \\
\text { Proteção Individual }\end{array}$ & $\begin{array}{l}\text { https://eaaf.org/wp- } \\
\text { content/uploads/covid1 } \\
\text { 9- } \\
\text { PDFs/Portugal/APTFerid } \\
\text { as-RECOMENDACAO- } \\
\text { PREPI-COVID19.pdf }\end{array}$ & $\begin{array}{l}\text { Orientação técnica da paramentação } \\
\text { dos EPIs, técnica de limpeza da pele } \\
\text { ante e após o uso dos EPIs, também } \\
\text { indica tipos de dispositivo e } \\
\text { coberturas para prevenir as lesões } \\
\text { cutâneas causadas pelos EPIs. }\end{array}$ & 4 \\
\hline 7 & $\begin{array}{l}\text { Jiang Q, Liu Y, Wei } \\
\text { W, Zhu D, Chen A, } \\
\text { Liu H, et al. }{ }^{21}\end{array}$ & $\begin{array}{l}\text { The prevalence, characteristics, } \\
\text { and related factors of pressure } \\
\text { injury in medical staff wearing } \\
\text { personal protective equipment } \\
\text { against COVID-19 in China: A } \\
\text { multicentre cross-sectional survey }\end{array}$ & $\begin{array}{l}\text { Int Wound J. } \\
\text { 2020;17(5):1300-9. doi: } \\
\text { 10.1111/iwj.13391 }\end{array}$ & $\begin{array}{l}\text { Prevalência das lesões de pele facial } \\
\text { causada pelos EPIs e os fatores de } \\
\text { risco para o profissional desenvolver } \\
\text { a lesão de pele facial. }\end{array}$ & 3 \\
\hline 8 & $\begin{array}{l}\text { Gefen A, Ousey } \\
\text { K. }{ }^{[22]}\end{array}$ & $\begin{array}{l}\text { Update to device-related pressure } \\
\text { ulcers: SECURE prevention. COVID- } \\
\text { 19, face masks and skin damage }\end{array}$ & $\begin{array}{l}\text { J Wound Care.2020; } \\
\text { 29(5):245-259. doi: } \\
\text { 10.12968/jowc.2020.29. } \\
5.245\end{array}$ & $\begin{array}{l}\text { Como prevenir as lesões de pele } \\
\text { facial causadas pelo uso dos EPIs. }\end{array}$ & 4 \\
\hline
\end{tabular}




\begin{tabular}{|c|c|c|c|c|c|}
\hline \multicolumn{2}{|r|}{ Autor } & \multirow{2}{*}{$\begin{array}{l}\text { Título } \\
\text { The preventive effect of } \\
\text { hydrocolloid dressing to prevent } \\
\text { facial pressure and facial marks } \\
\text { during use of medical protective } \\
\text { equipment in COVID-19 pandemic }\end{array}$} & \multirow{2}{*}{$\begin{array}{l}\text { Periódico/ano/volume } \\
\quad \text { /número } \\
\text { Br J Oral Maxillofac } \\
\text { Surg. 2020;58(6):723-5. } \\
\text { Citado em PMID: } \\
32418763\end{array}$} & \multirow{2}{*}{\begin{tabular}{l}
\multicolumn{1}{c}{ Resultados } \\
Descreve as técnicas e tipos de \\
coberturas que devem ser utilizados \\
para prevenir as lesões faciais \\
causadas pelo uso dos EPIs.
\end{tabular}} & \multirow{2}{*}{$\begin{array}{l}\text { Nível de } \\
\text { evidência } \\
3\end{array}$} \\
\hline 9 & $\begin{array}{l}\text { Del Castillo } \\
\text { Pardo de Vera } \\
\text { JL, Alcalde SR, } \\
\text { Carretero JLC, } \\
\text { García MB }{ }^{[23]}\end{array}$ & & & & \\
\hline 10 & $\begin{array}{l}\text { Ramalho AO, } \\
\text { Freitas PSS, } \\
\text { Nogueira PC. }{ }^{[24]}\end{array}$ & $\begin{array}{l}\text { Lesão por pressão relacionada a } \\
\text { dispositivo médico nos } \\
\text { profissionais de saúde em época } \\
\text { de pandemia }\end{array}$ & $\begin{array}{l}\text { Estima, Braz. J. } \\
\text { Enterostomal Ther., } \\
\text { 2020;18:e0120. doi: } \\
\text { 10.30886/ } \\
\text { estima.v18.867_PT }\end{array}$ & $\begin{array}{l}\text { Definição, fatores de risco, tipos de } \\
\text { coberturas para prevenir as lesões } \\
\text { de pele faciais causadas pelo uso } \\
\text { dos EPIs. } \\
\text { Limpeza da pele facial antes e após } \\
\text { uso dos EPIs para prevenir as lesões } \\
\text { causadas pelo uso dos EPIs. }\end{array}$ & 6 \\
\hline 11 & $\begin{array}{l}\text { Moore Z, Pattio } \\
\text { D, Avsar P, } \\
\text { McEvoy NL, } \\
\text { Gurley G, Budri } \\
\text { A, et al. }{ }^{[25]}\end{array}$ & $\begin{array}{l}\text { Prevention of pressure ulcers } \\
\text { among individuals cared for in the } \\
\text { prone position: lessons for the } \\
\text { COVID-19 emergenc }\end{array}$ & $\begin{array}{l}\text { JWound Care. 2020; } \\
\text { 29(6):312-20. doi: } \\
\text { 10.12968/jowc.2020.29. } \\
6.312\end{array}$ & $\begin{array}{l}\text { Oferece aos profissionais que } \\
\text { trabalham na sala de emergência as } \\
\text { medidas preventivas para lesões de } \\
\text { pele faciais causadas pelo uso dos } \\
\text { EPIs. }\end{array}$ & 4 \\
\hline 12 & Cruz EDA. ${ }^{[26]}$ & $\begin{array}{l}\text { Coping with the coronavirus - the } \\
\text { triad of reverse protection: by } \\
\text { protecting myself, I protect the } \\
\text { other, by protecting the other, I } \\
\text { protect myself }\end{array}$ & $\begin{array}{l}\text { Cogitare enferm. } \\
\text { 2020;25: e73708. doi: } \\
\text { 10.5380/ce.v25i0.73708 }\end{array}$ & $\begin{array}{l}\text { Enfrentamento do coronavírus - a } \\
\text { tríade da proteção reversa: ao me } \\
\text { proteger, protejo o outro, ao } \\
\text { proteger o outro, me protejo. }\end{array}$ & 4 \\
\hline 13 & $\begin{array}{l}\text { Oliveira AC, } \\
\text { Lucas TC, } \\
\text { lquiapaza RA. } \\
\text { [27] }\end{array}$ & $\begin{array}{l}\text { O que a pandemia da Covid-19 } \\
\text { tem nos ensinado sobre adoção } \\
\text { de medidas de precaução? }\end{array}$ & $\begin{array}{l}\text { Texto Contexto } \\
\text { Enferm.2020; } \\
\text { 29:e20200106. doi: } \\
\text { 10.1590/1980-265X- } \\
\text { TCE-2020-0106 }\end{array}$ & $\begin{array}{l}\text { Os cuidados que o profissional da } \\
\text { saúde e o paciente devem ter, } \\
\text { durante a pandemia do COVID-19, } \\
\text { para prevenir as lesões faciais e para } \\
\text { evitar a contaminação são: } \\
\text { higienização das mãos, uso dos EPIs, } \\
\text { uso do álcool em gel, etiqueta } \\
\text { respiratória, limpeza de superfícies, } \\
\text { evitar aglomerações e } \\
\text { distanciamento social. }\end{array}$ & 4 \\
\hline 14 & $\begin{array}{l}\text { Fumarola S, } \\
\text { Allaway R, } \\
\text { Callaghan R, } \\
\text { Collier M, } \\
\text { Downie F, } \\
\text { Geraghty J, et } \\
\text { al. }{ }^{[28]}\end{array}$ & $\begin{array}{l}\text { Overlooked and underestimated: } \\
\text { medical adhesive-related skin } \\
\text { injuries. Best practice consensus } \\
\text { document on preventio }\end{array}$ & $\begin{array}{l}\text { J Wound Care 2020; } \\
\text { 29(Suppl 3c):S1-S24. } \\
\text { doi: } \\
\text { 10.12968/jowc.2020.29. } \\
\text { Sup3c.S1 }\end{array}$ & $\begin{array}{l}\text { Após revisar a literatura, os autores } \\
\text { desenvolveram um instrumento } \\
\text { sobre lesões de pele relacionadas a } \\
\text { adesivos médicos e discutiram sobre } \\
\text { as melhores práticas para sua } \\
\text { avaliação e prevenção. }\end{array}$ & 4 \\
\hline 15 & $\begin{array}{l}\text { Padula CA, } \\
\text { Paradis HG, } \\
\text { Goodwin R, } \\
\text { Lynch J, } \\
\text { Hegerich- } \\
\text { Bartula D. }{ }^{[29]}\end{array}$ & $\begin{array}{l}\text { Prevention of injuries related to } \\
\text { medical devices associated with } \\
\text { the use of respiratory equipment } \\
\text { in a critical care unit: a quality } \\
\text { improvement project }\end{array}$ & $\begin{array}{l}\text { J Wound Ostomy } \\
\text { Continence Nurs. 2017; } \\
\text { 44(2):138-141. doi: } \\
\text { 10.1097/WON.0000000 } \\
000000311 \\
\text {. }\end{array}$ & $\begin{array}{l}\text { Oferece aos profissionais medidas } \\
\text { preventivas para lesões de pele } \\
\text { faciais causadas pelo uso dos EPIs } \\
\text { durante o uso dos EPIs }\end{array}$ & 4 \\
\hline 16 & $\begin{array}{l}\text { Oliveira HC, } \\
\text { Souza LC, Leite } \\
\text { TC, Campos } \\
\text { JF. V[30] }\end{array}$ & $\begin{array}{l}\text { Personal Protective Equipment in } \\
\text { the coronavirus pandemic: } \\
\text { training with Rapid Cycle } \\
\text { Deliberate Practice. }\end{array}$ & $\begin{array}{l}\text { Rev Bras Enferm. } \\
\text { 2020;73(Suppl } \\
\text { 2):e20200303. doi: } \\
\text { 10.1590/0034-7167- } \\
\text { 2020-0303 }\end{array}$ & $\begin{array}{l}\text { O enfrentamento da pandemia do } \\
\text { covid-19 requer uso apropriado de } \\
\text { Equipamento de Proteção Individual, } \\
\text { sendo importante que as } \\
\text { instituições forneçam treinamentos } \\
\text { aos profissionais quanto à } \\
\text { paramentação e desparamentação. }\end{array}$ & 4 \\
\hline 17 & $\begin{array}{l}\text { Sousa Neto AR } \\
\text { de, Freitas DRJ. } \\
\text { [31] }\end{array}$ & $\begin{array}{l}\text { Use of face masks: indications for } \\
\text { use and handling during the covid- } \\
19 \\
\text { Pandemic }\end{array}$ & $\begin{array}{l}\text { Cogitare enferm. 2020; } \\
\text { 25:e72867. } \\
\text { doi:10.5380/ce.v25i0.72 } \\
867\end{array}$ & $\begin{array}{l}\text { As máscaras devem ser utilizadas } \\
\text { durante a pandemia do COVID-19 } \\
\text { para prevenir a infecção e as lesões } \\
\text { de pele facial, porém os usuários } \\
\text { devem observar os seguintes } \\
\text { critérios: as máscaras devem estar } \\
\text { sempre limpas e secas. Utilizar papel } \\
\text { toalha para absorver a umidade, } \\
\text { usar creme hidratante antes e após } \\
\text { o uso da máscara. }\end{array}$ & 4 \\
\hline
\end{tabular}


Quadro 1. Características dos artigos selecionados por meio da revisão integrativa da literatura, para construção dos algoritmos. São Paulo, SP. Brasil, 2020 (conclusão)

\begin{tabular}{|c|c|c|c|c|c|}
\hline \multicolumn{2}{|r|}{ Autor } & \multirow{2}{*}{$\begin{array}{l}\text { Título } \\
\text { Reflections on recommendations } \\
\text { for the prevention of pressure } \\
\text { injuries during the Covid-19 } \\
\text { pandemic }\end{array}$} & \multirow{2}{*}{$\begin{array}{l}\text { Periódico/ano/volume } \\
\quad \text { /número } \\
\text { ESTIMA, Braz.J. } \\
\text { Enterostomal Ther. } \\
\text { 2020;18:e2520. doi: } \\
\text { 10.30886/estima.v18.94 } \\
\text { 0_PT }\end{array}$} & \multirow{2}{*}{$\begin{array}{l}\text { Resultados } \\
\text { Descreve os tipos de coberturas e } \\
\text { dispositivos para prevenir as lesões } \\
\text { de pele faciais causadas pelo uso } \\
\text { dos EPIs. }\end{array}$} & \multirow{2}{*}{$\begin{array}{l}\text { Nível de } \\
\text { evidência } \\
4\end{array}$} \\
\hline 18 & $\begin{array}{l}\text { Ramalho AO, } \\
\text { Freitas PSS, } \\
\text { Moraes JT, } \\
\text { Nogueira PC [32] }\end{array}$ & & & & \\
\hline 19 & $\begin{array}{l}\text { Gefen A, Alves } \\
\text { P, Ciprandi G, } \\
\text { Coyer F, Milne } \\
\text { CT, Ousey K et } \\
\text { al. }{ }^{[33]}\end{array}$ & $\begin{array}{l}\text { Device related pressure ulcers: } \\
\text { SECURE prevention. }\end{array}$ & $\begin{array}{l}\text { J Wound Care 2020; } \\
\text { 29(Sup2a): S1-S52 doi: } \\
\text { 10.12968/jowc.2020.29. } \\
\text { Sup2a.S1 }\end{array}$ & $\begin{array}{l}\text { Reafirma a importância de os } \\
\text { profissionais da saúde utilizarem os } \\
\text { EPIs, durante a pandemia do COVID- } \\
\text { 19, na prevenção das infecções e } \\
\text { das lesões de pele facial. }\end{array}$ & 4 \\
\hline 20 & $\begin{array}{l}\text { Coelho MMF, } \\
\text { Cavalcante } \\
\text { VMV, Moraes ]T, } \\
\text { Menezes LCG, } \\
\text { Figueirêdo SV, } \\
\text { Branco MFCC, } \\
\text { et al [34] }\end{array}$ & $\begin{array}{l}\text { Pressure injury related to the use } \\
\text { of personal protective equipment } \\
\text { in COVID-19 pandemic }\end{array}$ & $\begin{array}{l}\text { Rev Bras Enferm. } \\
\text { 2020;73(Suppl } \\
\text { 2):e20200670. doi: } \\
\text { 10.1590/0034-7167- } \\
\text { 2020-0670 }\end{array}$ & $\begin{array}{l}\text { Descreve a prevalência e medidas } \\
\text { preventivas para a lesão de pele } \\
\text { facial causada pelo uso dos EPIs. }\end{array}$ & 2 \\
\hline 21 & Cook TM. ${ }^{35}$ & $\begin{array}{l}\text { Equipamento de proteção } \\
\text { individual durante a pandemia da } \\
\text { doença coronavírus (COVID) } 2019 \\
\text { - uma revisão narrativa }\end{array}$ & $\begin{array}{l}\text { Anestesia. } \\
\text { 2020;75(7):920-927. } \\
\text { doi:10.1111/anae.15071 }\end{array}$ & $\begin{array}{l}\text { Ressalta a importância do uso dos } \\
\text { EPIs durante a pandemia do COVID- } \\
19 .\end{array}$ & 4 \\
\hline 22 & $\begin{array}{l}\text { Ong SWX, Tan } \\
\text { YK, Sutjipto S, } \\
\text { Chia PY, Young } \\
\text { BE, Gum M, et } \\
\text { al. [36] }\end{array}$ & $\begin{array}{l}\text { Absence of contamination from } \\
\text { personal protective equipment } \\
\text { (PPE) by severe acute respiratory } \\
\text { syndrome coronavirus } 2 \text { (SARS- } \\
\text { CoV-2). }\end{array}$ & $\begin{array}{l}\text { Infect Control Hosp } \\
\text { Epidemiol. } \\
\text { 2020;41(5):614-616. } \\
\text { doi:10.1017/ice.2020.91 }\end{array}$ & $\begin{array}{l}\text { Trata da importância de os } \\
\text { profissionais da saúde utilizarem os } \\
\text { EPIs, durante a pandemia do COVID- } \\
\text { 19, na prevenção das infecções e } \\
\text { das lesões de pele facial. }\end{array}$ & 3 \\
\hline 23 & Schank JE ${ }^{[37]}$ & $\begin{array}{l}\text { The NPUAP Meeting - This was No } \\
\text { Consensus Conference }\end{array}$ & $\begin{array}{l}\text { J Am Coll Clin Wound } \\
\text { Spec. 2016;7(1-3):19-24. } \\
\text { Published 2016 Jul } 28 . \\
\text { doi:10.1016/j.jccw.2016. } \\
07.001\end{array}$ & $\begin{array}{l}\text { Definição da lesão por pressão por } \\
\text { dispositivo médico. }\end{array}$ & 3 \\
\hline 24 & $\begin{array}{l}\text { Kelechi T], } \\
\text { Brunette G, Lee } \\
\text { LW [38] }\end{array}$ & $\begin{array}{l}\text { Personal Protective Equipment- } \\
\text { Related Equipment Dermatitis: A } \\
\text { View From Here }\end{array}$ & $\begin{array}{l}\text { J Wound Ostomy } \\
\text { Continence Nurs. 2020; } \\
\text { 47(4):324-5. doi: } \\
\text { 10.1097/WON.0000000 } \\
00000673\end{array}$ & $\begin{array}{l}\text { Discorre sobre a importância do uso } \\
\text { dos EPIs, durante a pandemia do } \\
\text { COVID-19, na prevenção de } \\
\text { dermatites. }\end{array}$ & 5 \\
\hline 25 & $\begin{array}{l}\text { Gondi S, } \\
\text { Beckman AL, } \\
\text { Deveau N, Raja } \\
\text { AS, Ranney ML, } \\
\text { Popkin R, et al. } \\
\text { [39] }\end{array}$ & $\begin{array}{l}\text { Personal protective equipment } \\
\text { needs in the USA during the } \\
\text { COVID-19 pandemic }\end{array}$ & $\begin{array}{l}\text { Lancet. 2020; } 395 \\
\text { (10237): e90-e91. doi: } \\
\text { 10.1016/ S0140-6736 } \\
\text { (20) 31038-2 }\end{array}$ & $\begin{array}{l}\text { Relata que a escassez dos EPIs } \\
\text { coloca em risco, tanto os pacientes } \\
\text { quanto os profissionais de saúde } \\
\text { durante a pandemia COVID-19. É } \\
\text { necessário que as instituições } \\
\text { forneçam os EPIs aos profissionais. } \\
\text { Quando o profissional utilizá-los } \\
\text { inadequadamente poderá contrair a } \\
\text { infecção e as lesões de pele } \\
\text { causadas pelo uso inadequado os } \\
\text { EPIs. }\end{array}$ & 4 \\
\hline 26 & $\begin{array}{l}\text { Atzrodt CL, } \\
\text { Maknojia I, } \\
\text { McCarthy RDP, } \\
\text { Oldfield TM, Po } \\
\text { J, Ta KTL, et al. } \\
\text { [40] }\end{array}$ & $\begin{array}{l}\text { A Guide to COVID-19: a global } \\
\text { pandemic caused by the novel } \\
\text { coronavirus SARS-CoV-2 }\end{array}$ & $\begin{array}{l}\text { FEBS J. 2020;287(17): } \\
\text { 3633-3650. Citado em: } \\
\text { PMID: } 32446285\end{array}$ & $\begin{array}{l}\text { Os profissionais de saúde correm } \\
\text { alto } \\
\text { risco de contrair a infecção, } \\
\text { especialmente quando usam os EPIs } \\
\text { inadequadamente. É importante que } \\
\text { as instituições proporcionem aos } \\
\text { profissionais da saúde treinamento } \\
\text { relacionado à paramentação e } \\
\text { desparamentação. }\end{array}$ & 4 \\
\hline
\end{tabular}

Fonte: Autoria própria. 


\section{Algoritmos}

Após a revisão integrativa da literatura, foram desenvolvidos três algoritmos, os quais descrevem as técnicas de paramentação, de desparamentação dos EPIs que os profissionais da saúde devem utilizar durante a pandemia da SARS-Cov-2 e as medidas para os profissionais da saúde se resguardarem das LPF causadas pelo uso inadequado dos EPIs na pandemia por SARS-CoV-2, conforme apresentado nas Figuras 2 a 4.

Figura 2. Algoritmo para orientar profissionais da saúde na utilização da técnica da paramentação dos equipamentos de proteção individual durante a pandemia da COVID-19. São Paulo, SP. Brasil, 2020

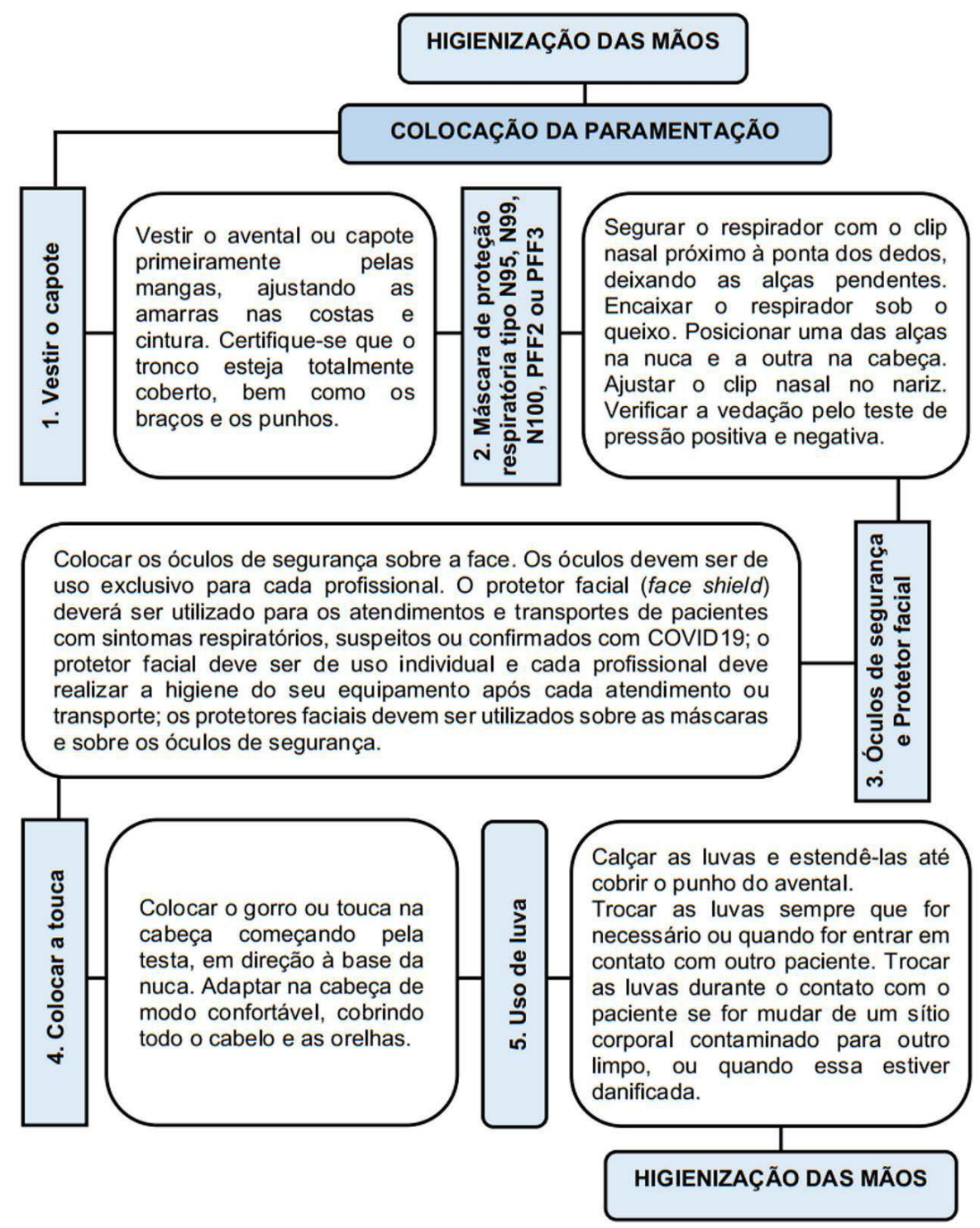

Fonte: Autoria própria. 


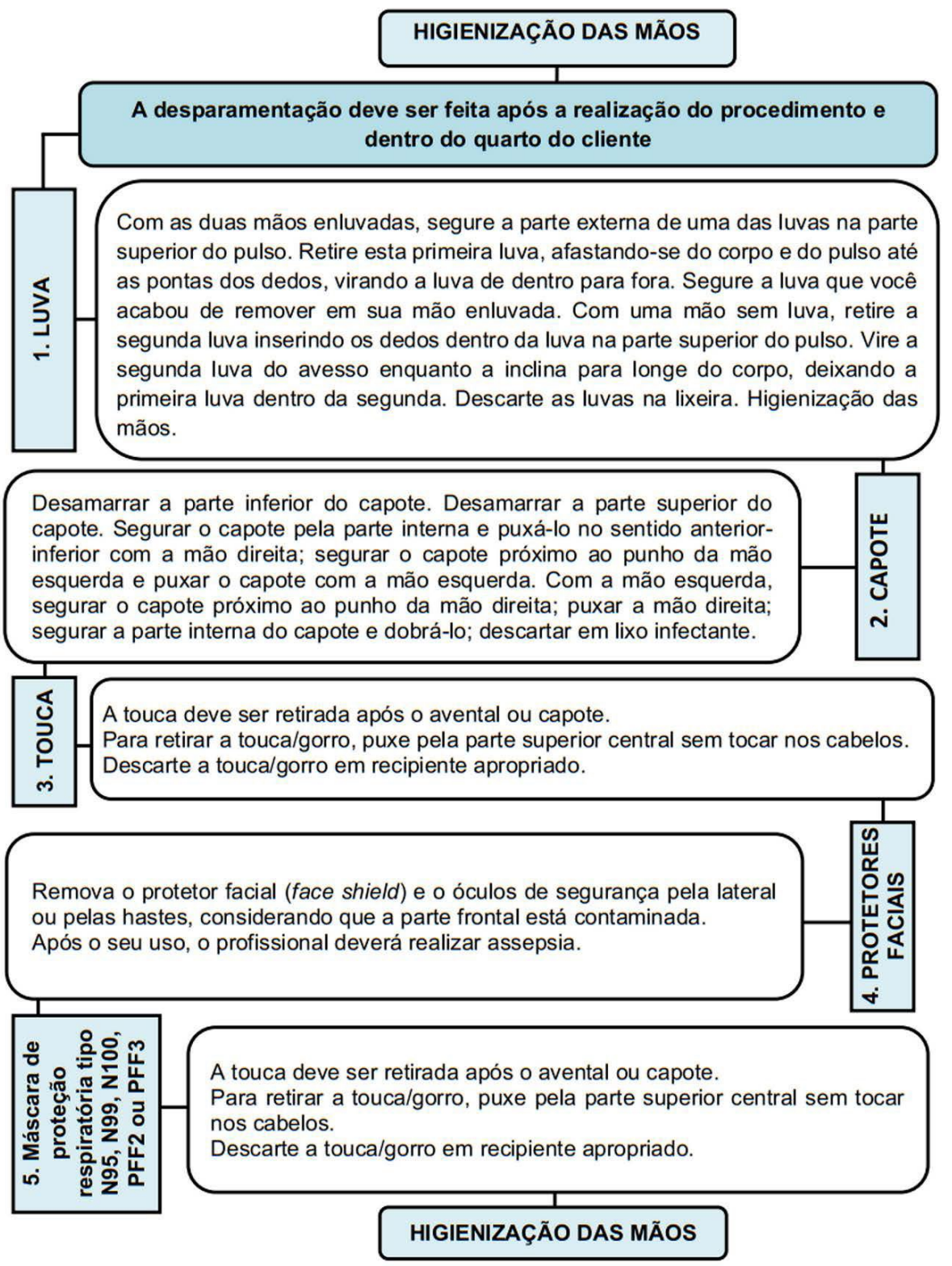

Fonte: Autoria própria. 


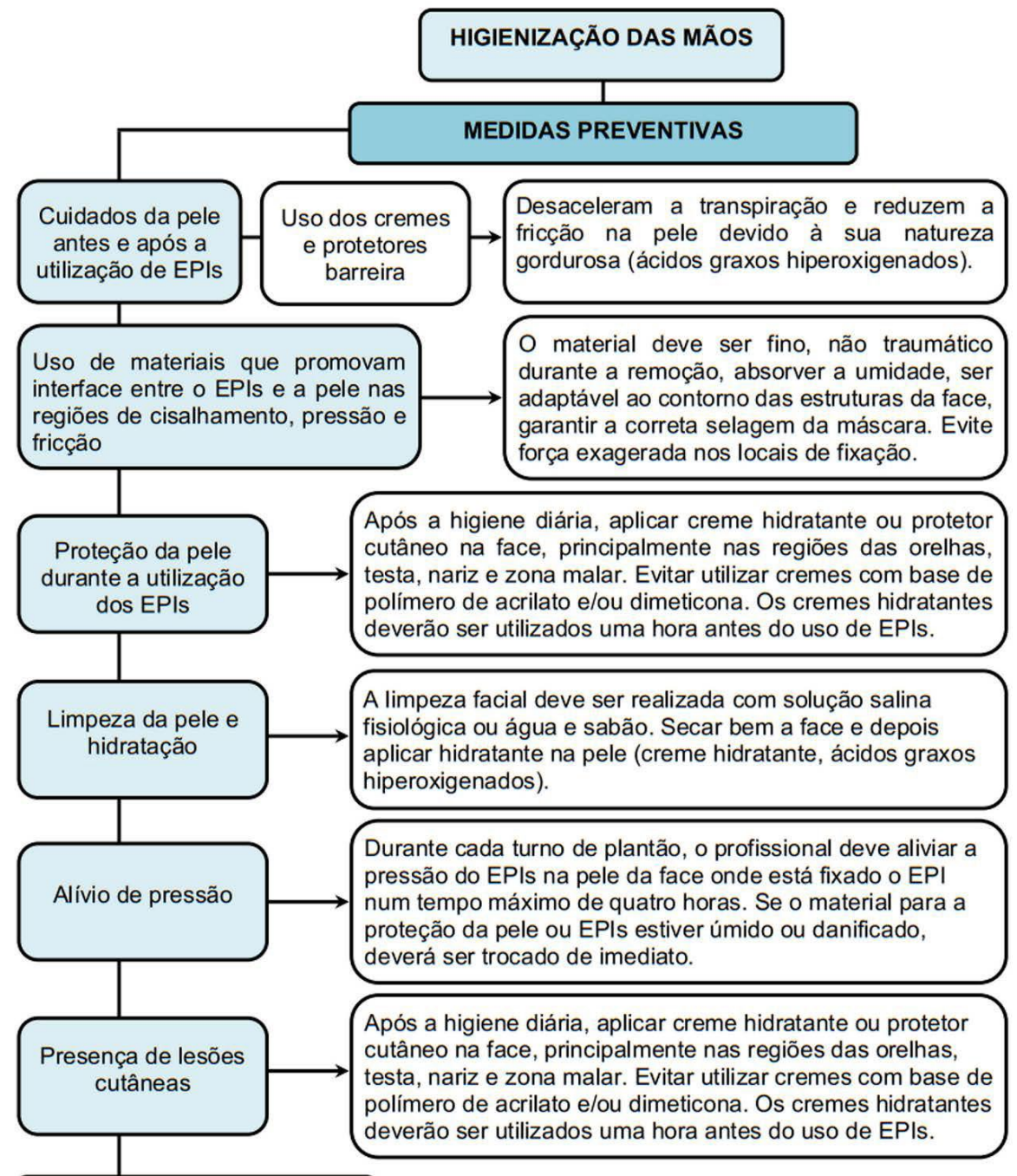

HIGIENIZAÇÃO DAS MÃOS

Fonte: Autoria própria. 


\section{Discussão}

Durante a pandemia da COVID-19, a segurança dos profissionais de saúde que estão na linha de frente está sendo assunto destacado pela mídia nacional e mundial. Vários profissionais estão contraindo a infecção e LPF por causa do uso inadequado dos EPIs; com isso, alguns profissionais estão com medo, estressados, com baixa autoestima e sentindo-se impotentes diante da doença. É necessário que as organizações de saúde ofereçam os EPIs e dispositivos para prevenir as lesões e, principalmente, desenvolvam protocolos e treinamento com a finalidade de promover um cuidado livre de danos e eventos adversos para o profissional.

Os profissionais de saúde que estão na linha de frente ao combate à COVID-19 mostram exaustão física e mental, dificuldades na tomada de decisão e ansiedade pela dor em perder pacientes e colegas, além do risco de infecção e das LPF e a possibilidade de transmitir para familiares. Assim, garantir a assistência de saúde para os profissionais da área de saúde, os EPIs e apoio psicológico são fundamentais. 5,40 Assim como desenvolver protocolos, oferecer treinamento relacionado à técnica do uso dos EPIs e medidas preventivas para LPF.,, 21

Padronização de protocolos e treinamento durante a pandemia da COVID-19 possibilitam ao profissional relembrar de como efetivar a técnica, que tem como consequência assistência sem danos e principalmente a diminuição de risco em adquirir infecção e LPF causado pelo uso inadequado dos EPIs.

Neste estudo, foram desenvolvidos três algoritmos com o objetivo de oferecer as técnicas da paramentação, de desparamentação dos EPIs e as medidas preventivas para prevenir as lesões causadas pelo uso inadequado dos EPIs. O desenvolvimento de um algoritmo deve ser realizado com embasamento científico, incluindo artigos com evidências clínicas, com a finalidade de auxiliar nos procedimentos técnicos, clínicos, administrativos e financeiros, com o objetivo de prevenir infecção, melhorar a assistência e diminuir o custo do tratamento. $\frac{10,11}{11}$

A adoção, padronização e orientação aos profissionais sobre a técnica do uso dos EPIs, principalmente durante a pandemia da COVID-19, são fundamentais na proteção e prevenção dos efeitos adversos.

O profissional da saúde que está na linha de frente na pandemia do COVID-19 encontra-se em risco aumentado de exposições da infecção, esse risco eleva-se diante da necessidade frequente de realizar procedimentos invasivos e ampla gama de distúrbios que o paciente apresenta, sendo necessário observar as precauções padronizadas pela Organização Mundial da Saúde. Visando minimizar o risco, também se recomenda que as organizações hospitalares forneçam EPIs e treinamentos da utilização da paramentação e desparamentação dos EPIs. 3,18,41-46

Compreende-se como EPIs touca, aventais, luvas, óculos protetores, protetor facial, máscaras e o seu uso deve ser rotineiro e incorporado ao cotidiano do profissional. Os referidos dispositivos de proteção constituem materiais básicos durante a pandemia do COVID-19, necessários e indispensáveis para evitar a disseminação de infecção no ambiente hospitalar, bem como para manter e proteger sua integridade física e previne as LPF, uma vez que, neste ambiente, há maior possibilidade e facilidade de se contrair patologias, pela peculiaridade do local e função/atividade exercida. $.18,19,43,47$

O Algoritmo para prevenir lesão causada pelo uso dos equipamentos de proteção individual na pandemia da COVID-19 fornece aos profissionais as ações preventivas para prevenir as LPF, as quais devem ser incorporadas às rotinas dos profissionais da saúde, no que se refere à higiene da pele e utilização de creme hidratante antes e depois do uso dos EPIs, e recomenda o uso de coberturas para prevenir a LPF.

Uma lesão de pele relacionada ao uso de dispositivos (máscara, óculos, protetor facial) é considerada aquela que ocasiona irritação, ferida e eritema que permanece por mais de 30 minutos após sua remoção. Quando os dispositivos permanecem úmidos, podem ocasionar a maceração na pele, o que contribui para a ocorrência de lesões. Esse tipo de lesão acontece quando a força do dispositivo é superior à da pele, e acaba por separar a epiderme da derme, que pode causar dor e oferecer risco de infecção, prejudicando a qualidade de vida do profissional.22,25,48,49 
No que tange às medidas de prevenção e de tratamento das LPF, existem as gerais que incluem a higienização da pele com sabonete de preferência com pH compatível com a pele (levemente acidificado), a hidratação da pele com creme hidratante sem presença de lipídios, que deve ser realizada antes e após a retirada dos dispositivos. O profissional também deve aplicar uma cobertura profilática como interface entre a pele e a área de fixação dos dispositivos, que pode ser com coberturas de espuma de poliuretano, silicone, filme transparente ou placa de hidrocoloide extrafino. $24,37-39,50$

\section{Conclusão}

Os algoritmos construídos oferecem ao profissional de saúde a descrição de técnicas do uso dos EPIs, medidas preventivas para LPF causadas pelo uso dos EPIs, assim como possibilitam ao profissional uma melhor visualização, praticidade e entendimento do procedimento a ser realizado.

\section{Conflitos de interesses}

Nenhum conflito financeiro, legal ou político envolvendo terceiros (governo, empresas e fundações privadas, etc.) foi declarado para nenhum aspecto do trabalho submetido (incluindo, mas não se limitando a subvenções e financiamentos, participação em conselho consultivo, desenho de estudo, preparação de manuscrito, análise estatística, etc.).

\section{Referências}

1. World Health Organization. Coronavirus disease 2019 (COVID-19) Situation report -87 [Internet]. 2020 [citado 2020 Mai 02]. Disponível em: https://www.who.int/docs/default-source/ coronaviruse/situation-reports/20200416-sitrep-87-covid-19. pdf?sfvrsn=9523115a_2

2. Freitas BHBM, Alves MDSM, Gaíva MAM. Medidas de prevenção e controle de infecção neonatal por COVID-19: revisão de escopo. Rev Bras Enferm. 2020;73(Supl 2):e20200467. http://dx.doi. org/10.1590/0034-7167-2020-0467

3. Baig AM, Khaleeq A, Ali U, Syeda H. Evidence of the COVID-19 Virus Targeting the CNS: Tissue Distribution, Host-Virus Interaction, and Proposed Neurotropic Mechanisms. ACS Chem Neurosci. 2020;11(7):995-8. https://doi.org/10.1021/ acschemneuro.0c00122
4. Deng C. The global battle against SARS-CoV-2 and COVID-19. Int J Biol Sci. 2020;16(10):1676-7. https://doi.org/10.7150/ijbs.45587

5. Medeiros EA. A luta dos profissionais de saúde no enfrentamento da COVID-19. Acta Paul Enferm. 2020;33:e-EDT20200003. http://dx.doi.org/10.37689/actaape/2020EDT0003

6. Wiwanitkit V. N95 respirator, COVID-19, and health care worker. Int Wound J. 2020;17(5):1534. Citado em: PMID: 32488933

7. Holland M, Zaloga DJ, Friderici CS. COVID-19: Personal Protective Equipment (PPE) for the emergency physician. Vis J Emerg Med. 2020;19:100740. https://doi.org/10.1016/j. visj.2020.100740

8. Lam UN, Md Mydin Siddik NSF, Mohd Yussof SJ, Ibrahim S. N95 respirator associated pressure ulcer amongst COVID-19 health care workers. Int Wound J. 2020;17(5):1525-1527. Citado em: PMID: 32397010

9. World Health Organization. Rational use of personal protective equipment (PPE) for coronavirus disease (COVID-19). Interim guidance [Internet]. 2020. [Citado 2020 Jul 15]. Disponível em: https://www.who.int/publications/i/item/rational-use-of-personalprotective-equipment-for-coronavirus-disease-(covid-19)-andconsiderations-during-severe-shortages

10. Carvalho MRF, Salomé GM, Ferreira LM. Construção e validação de algoritmo para tratamento da lesão por pressão. J Nurs UFPE on line [Internet]. 2017;11(Sup 10):4171-83. Disponível em: https://periodicos.ufpe.br/revistas/revistaenfermagem/ article/view/231180

11. Salomé GM, Rocha CA, Miranda FD, Alves JR, Dutra RAA, Tenório AG. Algoritmos para prevenção e tratamento de dermatite associada à incontinência. Estima. 2020;18(1):e1320. https://doi.org/10.30886/estima.v18.837_PT

12. Mendes KDS, Silveira RCPC, Galvão CM. Revisão integrativa: método de pesquisa para a incorporação de evidências na saúde e na enfermagem. Texto Contexto Enferm. 2008;17(4):758-64. https://doi.org/10.1590/S0104-07072008000400018

13. Santos CMC, Pimenta CAM, Nobre MRC. A estratégia PICO para a construção da pergunta de pesquisa e busca de evidências. Rev Latino-Am Enfermagem. 2007;15(3):508-11. https://doi. org/10.1590/S0104-11692007000300023

\begin{abstract}
14. Agency for Health Care Research and Quality. Quality Improvement and monitoring at your fingertips [Internet]. Rockville: Agency for Healthcare Research and Quality; 2016 [citado em 2019 mar 22]. Disponível em: http://www. qualityindicators.ahrq.gov
\end{abstract}

15. Black J. COVID-19 and wound care in the US. Wounds International [Internet]. 2020;11(2):6-7. https://www. woundsinternational.com/resources/details/covid-19-and-woundcare-us 
16. Taminato M, Mizusaki-Imoto A, Saconato H, Franco ES. N95 respirator associated pressure ulcer amongst COVID-19 health care workers. Int Wound J. 2020;17(5):1525-27. https://doi. org/10.1111/iwj.13398

17. Taminato M, Mizusaki-Imoto A, Saconato H, Franco EB, Puga ME, Duarte ML, et al. Mascaras de tecido na contenção de gotículas respiratórias - revisão sistemática. Acta Paul Enferm. 2020;33:eAPE20200103. http://dx.doi.org/10.37689/ actaape/2020AR0103

18. Gefen A, Ousey K. Prevention of skin damage caused by the protective equipment used to mitigate COVID-19. J Wound Care. 2020;29(6):311. Citado em: PMID: 32530779

19. Smart H, Opinion FB, Darwich I, Elnawsany MA, Kodange C. Preventing Facial Pressure Injury for Health Care Providers Adhering to COVID-19 Personal Protective Equipment Requirements. Adv Skin Wound Care. 2020;33(8):418-27. https:// doi.org/10.1097/01.asw.0000669920.94084.c1

20. Alves $P$, Moura A, Vaz A, Ferreira A, Malcato E, Mota S, et al. Recomendação PREPI | COVID19. Prevenção de lesões cutâneas causadas pelos Equipamentos de Proteção Individual (Máscaras faciais, respiradores, viseiras e óculos de proteção). Journal of Tissue Healing and Regeneration. 2020. Suplemento da edição Outubro/Março XV. Disponível em: https://eaaf.org/wp-content/ uploads/covid19-PDFs/Portugal/APTFeridas-RECOMENDACAOPREPI-COVID19.pdf

21. Jiang Q, Liu Y, Wei W, Zhu D, Chen A, Liu H, et al. The prevalence, characteristics, and related factors of pressure injury in medical staff wearing personal protective equipment against COVID-19 in China: A multicentre cross-sectional survey. Int Wound J. 2020;17(5):1300-9. https://doi.org/10.1111/iwj.13391

22. Gefen A, Ousey K. Update to diveci-related pressure ulcer: segure prevention COVID-19, face masks and skin damage. J Wound Care. 2020;29(5):245-59. http://dx.doi.org/10.12968/ jowc.2020.29.5.245

23. Del Castillo Pardo de Vera JL, Alcalde SR, Carretero JLC, García $M B$. The preventive effect of hydrocolloid dressing to prevent facial pressure and facial marks during use of medical protective equipment in COVID-19 pandemic. Br J Oral Maxillofac Surg. 2020;58(6):723-5. Citado em: PMID: 32418763

24. Ramalho AO, Freitas PSS, Nogueira PC. Lesão por pressão relacionada a dispositivo médico nos profissionais de saúde em época de pandemia. ESTIMA, Braz. J. Enterostomal Ther. 2020;18:e0120. https://doi.org/10.30886/estima.v18.867_PT

25. Moore Z, Pattio D, Avsar P, McEvoy NL, Gurley G, Budri A, et al. Prevention of pressure ulcers among individuals cared for in the prone position: lessons for the COVID-19 emergency. J Wound Care. 2020;29(6):312-20. https://doi.org/10.12968/ jowc.2020.29.6.312
26. Cruz EDA. Enfrentamento do coronavírus - a tríade da proteção reversa:ao me proteger, protejo o outro ao proteger o outro, me protejo. Cogitare enferm. 2020;25: e73708. http:// dx.doi.org/10.5380/ce.v25i0.73708

27. Oliveira AC, Lucas TC, Iquiapaza RA. O que a pandemia da Covid-19 tem nos ensinado sobre adoção de medidas de precaução?. Texto contexto - Enferm. 2020;29:e20200106. https:// doi.org/10.1590/1980-265x-tce-2020-0106

28. Fumarola S, Allaway R, Callaghan R, Collier M, Downie F, Geraghty J, et al. Overlooked and underestimated: medical adhesive-related skin injuries. Best practice consensus document on prevention. J Wound Care. 2020;29(Suppl 3c):S1-S24. https:// doi.org/10.12968/jowc.2020.29.Sup3c.S1

29. Padula CA, Paradis H, Goodwin R, Lynch J, Hegerich-Bartula D. Prevention of Medical Device-Related Pressure Injuries Associated With Respiratory Equipment Use in a Critical Care Unit: A Quality Improvement Project. J Wound Ostomy Continence Nurs. 2017;44(2):138-41. https://doi.org/10.1097/ won.0000000000000311

30. Oliveira HC, Souza LC, Leite TC, Campos JF. Personal Protective Equipment in the coronavirus pandemic: training with Rapid Cycle Deliberate Practice. Rev Bras Enferm. 2020;73(Suppl 2):e20200303. http://dx.doi.org/10.1590/0034-7167-2020-0303

31. Sousa Neto AR, Freitas DRJ. Utilização de máscaras: indicações de uso e manejo durante a pandemia de Covid-19. Cogitare enferm. 2020;25:e72867. http://dx.doi.org/10.5380/ce.v25i0.72867

32. Ramalho AO, Freitas PSS, Moraes JT, Nogueira PC. Reflections on recommendations for the prevention of pressure injuries during the Covid-19 pandemic. ESTIMA, Braz. J. Enterostomal Ther. 2020;18:e2520. https://doi.org/10.30886/estima.v18.940_PT

33. Gefen A, Alves P, Ciprandi G, Coyer F, Milne CT, Ousey K et al Device related pressure ulcers: SECURE prevention. J Wound Care. 2020; 29(Sup2a): S1-S52. https://doi.org/10.12968/jowc.2020.29. Sup2a.S1

34. Coelho MMF, Cavalcante VMV, Moraes JT, Menezes LCG, Figueirêdo SV, Branco MFCC, et al. Pressure injury related to the use of personal protective equipment in COVID-19 pandemic. Rev Bras Enferm. 2020;73(Suppl 2):e20200670. http:// dx.doi.org/10.1590/0034-7167-2020-0670

35. Cook TM. Personal protective equipment during the coronavirus disease (COVID) 2019 pandemic - a narrative review. Anestesia. 2020;75(7):920-927. https://doi.org/10.1111/ anae.15071

36. Ong SWX, Tan YK, Sutjipto S, Chia PY, Young BE, Gum M, et al. Absence of contamination of personal protective equipment (PPE) by severe acute respiratory syndrome coronavirus 2 (SARS-CoV-2). Infect Control Hosp Epidemiol. 2020;41(5):614-6. https://doi. org/10.1017/ice.2020.91 
37. Schank JE. The NPUAP Meeting - This was No Consensus Conference. J Am Coll Clin Wound Spec. 2016;7(1-3):19-24. https:// doi.org/10.1016/j.jccw.2016.07.001

38. Kelechi TJ, Brunette G, Lee LW. Personal Protective Equipment-Related Equipment Dermatitis: A View From Here. J Wound Ostomy Continence Nurs. 2020;47(4):324-5. https://doi. org/10.1097/won. 0000000000000673

39. Gondi S, Beckman AL, Deveau N, Raja AS, Ranney ML, Popkin $\mathrm{R}$, et al. Personal protective equipment needs in the USA during the COVID-19 pandemic. Lancet. 2020;395(10237):e90-e91. https://doi.org/10.1016/s0140-6736(20)31038-2

40. Atzrodt CL, Maknojia I, McCarthy RDP, Oldfield TM, Po J, Ta KTL, et al. A Guide to COVID-19: a global pandemic caused by the novel coronavirus SARS-CoV-2. FEBS J. 2020;287(17): 3633-3650. Citado em: PMID: 32446285

41. Ferioli M, Cisternino C, Leo V, Pisani L, Palange $P$, Nava S. Protecting healthcare workers from SARS-CoV-2 infection: practical indications. Eur Respir Rev. 2020;29(155):20006. Citado em: PMID: 32248146

42. Rieth GH, Loro MM, Stumm EMF, Rosanelli CLSP, Kolankiewicz ACB, Gomes JS. Uso de equipamentos de proteção individual pela enfermagem em uma unidade de emergência hospitalar. J Nurs UFPE on line. 2014;8(2):365-71. https://doi.org/10.5205/ reuol.4688-38583-1-RV.0802201418

43. Barbosa JAG, Souza MCMR. Lesões de pele relacionadas ao uso de adesivos: cuidados para sua prevenção como condição para assistência segura e de qualidade. Revista Recien [Internet]. 2020; 10(29):13-18. https://doi.org/10.24276/ rrecien2358-3088.2020.10.29.13-18
44. Galindo Neto NM, Sá GGM, Barbosa LU, Pereira JCN, Henriques AHB, Barros LM. Covid-19 and digital technology: mobile applications available for download in smartphones. Texto Contexto Enferm. 2020;29:e20200150. https://doi. org/10.1590/1980-265X-TCE-2020-0150

45. Santana RF, Silva MB, Marcos DASR, Rosa CS, Wetzel Jr W, Delvalle R. Nursing recommendations for facing dissemination of COVID-19 in Brazilian Nursing Homes. Rev Bras Enferm. 2020;73(Suppl 2):e20200260. http://dx.doi.org/10.1590/00347167-2020-0260

46. Apaijitt $P$, Wiwanitkit V. Knowledge of coronavirus disease 2019 (COVID-19) by medical personnel in a rural area of Thailand. Infect Control Hosp Epidemiol. 2020;41(10):1243-44. https://doi. org/10.1017/ice.2020.159

47. Nazerali RS, Song KR, Wong MS. Facial pressure ulcer following prone positioning. J Plast Reconstr Aesthet Surg. 2010;63(4): e413-4. Citado em: PMID: 19939757

48. Salomé GM, Pontes BCD. Lesões por pressão durante a pandemia da covid-19. J Nurs UFPE on line. 2021;15:e241981. https://doi.org/10.5205/1981-8963.2021.246189

49. Yıldız A, Karadağ A, Yıldız A, Çakar V. Determination of the effect of prophylactic dressing on the prevention of skin injuries associated with personal protective equipments in health care workers during COVID-19 pandemic. J Tissue Viability. 2021;30(1):21-27. Citado em: PMID: 33187790

50. Tabah A, Ramanan M, Laupland KB, Buetti N, Cortegiani A, Mellinghoff J, et al. Personal protective equipment and intensive care unit healthcare worker safety in the COVID-19 era (PPE-SAFE): An international survey. J Crit Care. 2020;59:70-75. Citado em: PMID: 32570052 